\title{
ancres marines - ancrage à terre
}

\author{
par \\ P. Habib \\ Laboratoire de Mécanique des Solides (E.P. - ENSM Paris - ENPC - ERA CNRS) \\ P. Le Tirant \\ Institut Français du Pétrole (Rueil Malmaison) \\ M. P. Luong \\ Laboratoire de Mécanique des Solides (E.P. - ENSM Paris - ENPC - ERA CNRS)
}

\begin{abstract}
Les travaux qui sont présentés ici sont le fruit d'une collaboration entre le Laboratoire de Mécanique des Solides, I'Institut Français du Pétrole et les Constructions Métalliques de Provence. Cette étude effectuée en commun par un laboratoire de recherches fondamentales, par un laboratoire de recherches appliquées et par une industrie, pour la mise en œuvre d'un produit nouveau, à savoir une ancre, est actuellement en cours de développement. Cependant, il a semblé que des résultats suffisamment intéressants pour pouvoir être présentés avaient déjà été obtenus. MM. Puech (I. F.P.) et Perrin (C.M.P.) ont participé à ces travaux pour la conception et la réalisation de certains prototypes ainsi que pour leur expérimentation.
\end{abstract}

\section{Introduction}

Les ancres marines sont des objets qui ont été étudiés essentiellement par des marins (J. Le Roy, 1972) et il y a peu de temps que des spécialistes de mécanique des sols se sont véritablement intéressés à ce problème (Puech, Meunier et Paillard, 1978), dans le but d'évaluer les qualités de matériels qui ont évolué progressivement dans un grand empirisme pour arriver aux modèles actuels. Les problèmes que posent aux marins les ancrages traditionnels sont d'ailleurs bien éloignés des soucis du géotechnicien. Le marin ne connaît pas toujours la nature du fond où il veut mouiller, mais le connaitrait-il qu'il ne disposerait probablement pas de la gamme des ancres spécifiques à chaque sol. Le géotechnicien par contre ne sait ni le vent ni la mer et son premier réflexe est de chercher à reconnaître et définir le sol du fond de la mer pour tirer au mieux parti de ses qualités : deux mondes étrangers qui n'avaient aucune chance de se rencontrer avant les problèmes nouveaux et difficiles posés par les travaux pétroliers en haute mer. Rappelons d'abord ce qu'est une ancre.

\section{Les ancres marines}

Une ancre marine est un élément d'un ensemble destiné à retenir un bateau soumis à la force du vent ou du courant. Pour cela une ligne est fixée au bateau et elle est constituée d'un câble, d'une chaîne et d'une ancre ou bien seulement d'une chaîne et d'une ancre (fig. 1). Une partie de la chaine reste sur le fond selon la tension de la ligne. Sous l'effet de son poids, la chaîne dans l'eau prend la forme classique de la chaînette; la force horizontale d'ancrage est ainsi donnée par l'ancre et par un morceau de chaîne qui traîne sur le sol et dont la longueur dépend de la résistance de l'ancre, c'est-à-dire aussi du sol. La chaîne est un organe très important, qui fait l'objet de calculs attentifs. Pour

Fig. 1 Principe de l'ancre marine

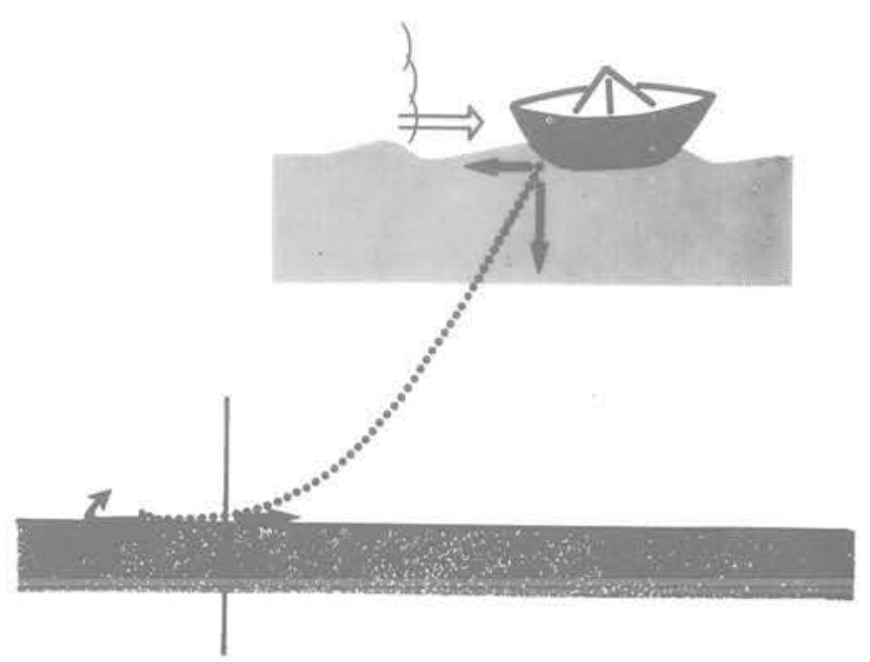


donner des ordres de grandeur, on peut mettre $40 \mathrm{~kg}$ de chaîne à une ancre de plaisance de $12 \mathrm{~kg}$; à une ancre un peu exceptionnelle de $35 \mathrm{t}$, pour un grand pétrolier, on peut associer $200 \mathrm{t}$. de chaine. Il existe des règles pour fixer la longueur et la dimension des chaînes, qui sont fonction de la nature et de la profondeur du mouillage, de la taille du navire, de la nuance de l'acier de la chaîne (dont dépend le poids des maillons) : c'est une véritable structure et on ne peut pas la négliger.

La première ancre, dite ancre phénicienne, semble avoir été une grosse pierre trouée, attachée à une corde et jetée au fond de l'eau : c'était un véritable corps mort. Posée sur un sol pulvérulent dont l'angle de frottement en surface est de l'ordre de $35^{\circ}$ et soumise à l'action d'une force horizontale $T$, cette ancre, dont le poids déjaugé est $P$, dérape lorsque $T$ atteint $0,7 \mathrm{P}$ : la résistance de l'ancrage est ainsi proportionnelle au poids de l'ancre.

La figure 2 indique la résistance du corps mort sous l'effet d'une traction qui s'incline jusqu'à l'horizontale,

Lorsque ce corps mort est posé sur un fond d'argile, il y a encore proportionnalité entre le poids et la résistance. En effet, la pression de contact au sol est limitée par la résistance à l'écrasement des sommets des inégalités du fond soit: $p=(2+\pi) c$ (fig. 3). La surface de contact est donc $S=\frac{P}{(2+\pi) c}$. Or, la résistance au glissement latéral est $\mathrm{T}=\mathrm{cS}$, d'où $T=\frac{P}{2+\pi} \# 0,2 P$. En fait, il s'agit là d'un ordre de grandeur, car la couche superficielle de l'argile du fond est peut-être moins résistante, ce qui peut diminuer $\mathrm{T}=\mathrm{c}^{\prime} \mathrm{S}$ de moitié, ou bien le contact au sol du corps mort peut être plus grand ce qui peut aller jusqu'à doubler $\mathrm{T}=\mathrm{cS}^{\prime}$. Mais si T est beaucoup plus grand que $\frac{\mathrm{P}}{2}$ cela signifie qu'il y a eu poinçonnement et apparition d'un effet de butée (à droite sur la fig. 3). Montrons que la résistance due à la butée est aussi fonction du poids de l'ancre. Imaginons un écran vertical soumis à une force horizontale $\mathrm{H}$ (fig. $4 \mathrm{a}$ ); au-delà de la résistance limite l'écoulement plastique engendre un glissement incliné qui fait monter l'écran et le coin de terre associé. Si on veut empêcher ce mouvement, il est nécessaire d'ajouter une force verticale $V$, ce qui revient à incliner vers le bas la force qui agit sur l'écran, de telle façon que la ligne marginale du réseau d'écoulement plastique ait une tangente horizontale au bord inférieur de l'écran (fig. 4 b). En réalité, la profondeur d'enfouissement de l'ancre se règle en

Fig. 4a Butée horizontale sur un écran vertical

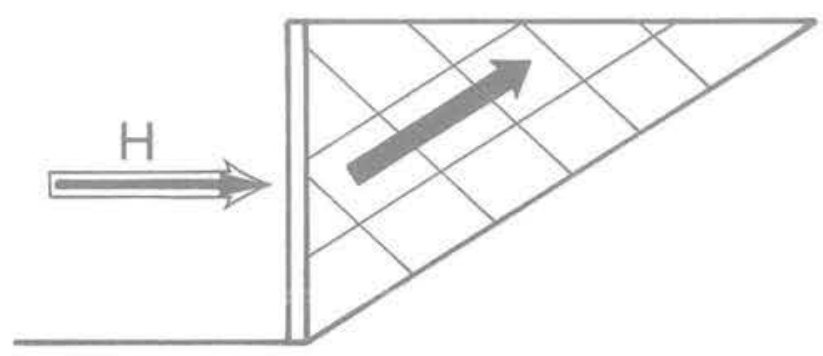

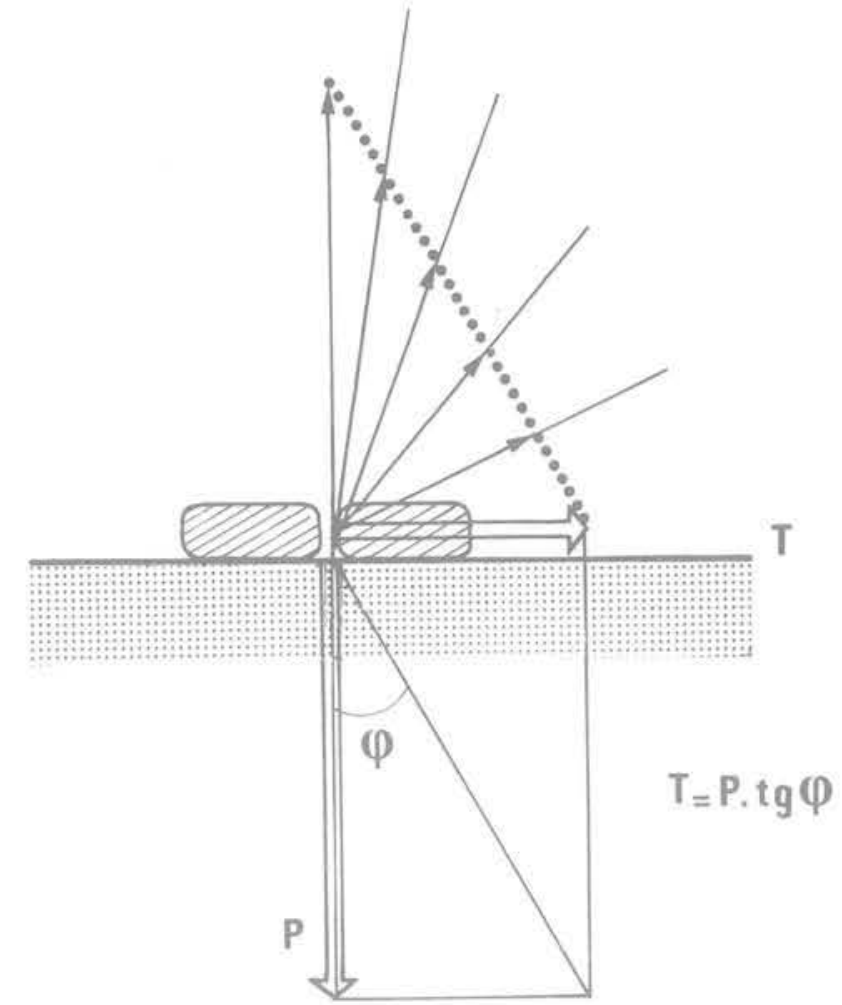

Fig. 2 Corps mort sur fond de sable

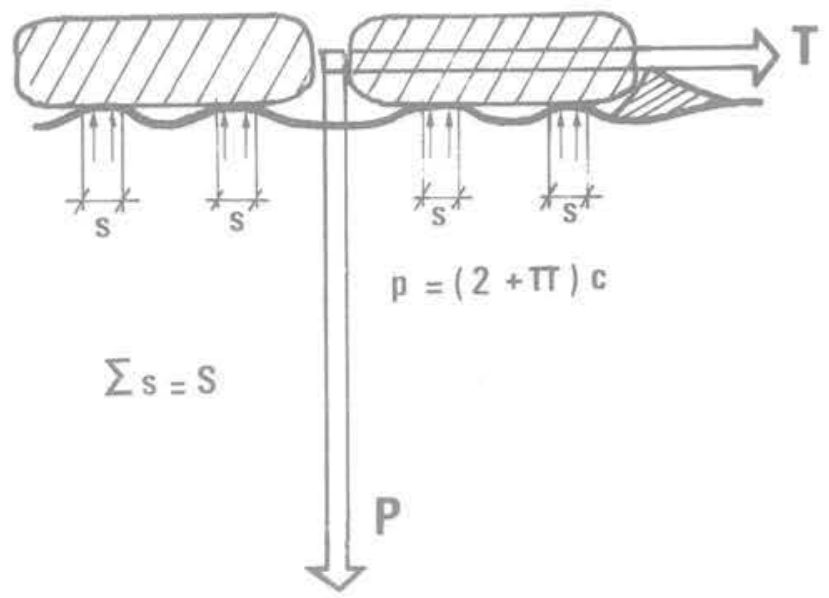

Fig. 3 Corps mort sur fond argileux

Fig. $4 b$ Butée inclinée pour un déplacement horizontal

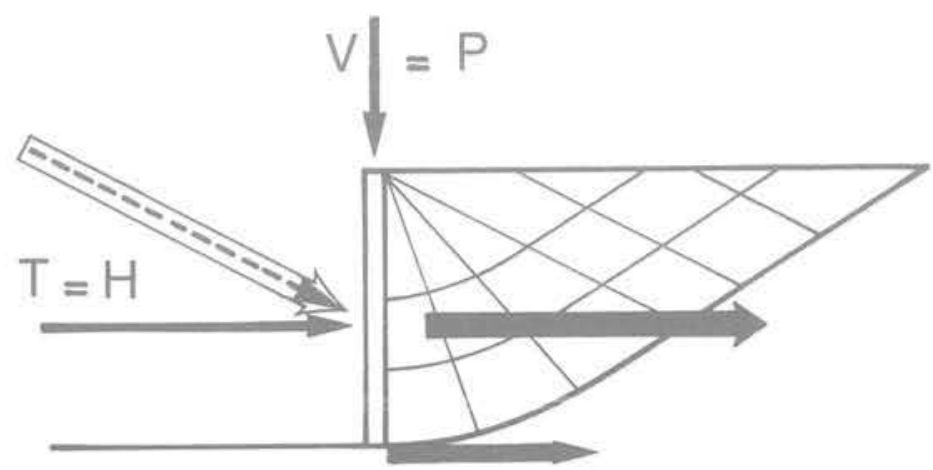


fonction de son poids. Si l'ancre est placée trop profond, elle aura tendance à ressortir; si elle est placée trop en surface, elle aura tendance à s'enfoncer au cours du dragage. La profondeur de pénétration, donc la résistance de butée de l'ancre, est encore fonction du poids.

L'écran agissant comme un ancrage présente un inconvénient: pour mobiliser sa résistance, il faut l'orienter préalablement dans une direction perpendiculaire à l'azimut de la traction, alors que la pierre des phéniciens résistait dans toutes les directions. Pour obtenir un bon résultat, quelle que soit l'orientation de la traction, il faut donc faire trainer l'ancre pour qu'elle se dispose convenablement.

La classique ancre à jas (fig. 5 ) réalise parfaitement ce mécanisme, mais elle profite en plus de l'effet tridimensionnel de l'ancrage sur la pointe. Le jas maintient l'ancre dans la bonne position (fig. 6) quand on la tire par l'œil; la verge permet une orientation correcte; la patte s'enfonce au cours du mouvement sous l'effet du poids propre et selon la nature du fond; la pointe mobilise la résistance à l'arrachement d'une forme cônique dont le sommet se trouve au bout de la patte ainsi que d'un drièdre qui prend appui sur le corps de la patte, ce qui évidemment est très avantageux si on compare à un problème à deux dimensions. On constate ainsi que l'ancre n'exerce toute sa résistance qu'après un certain déplacement qui correspond d'abord à son orientation, ensuite à son enfouissement.

L'ancre à jas est un engin un peu ancien. A partir de 1820 , on a commencé à construire des ancres avec des pattes articulées (fig. 7), qui se déployaient lors de la mise en place : se sont les ancres dites réversibles (fig. 8 et 9 ). $I I$ en existe de très nombreux modèles (Hall, Manheim, Byers, Stokes, etc.). Sur ces ancres classiques, un empirisme de plusieurs générations a permis d'améliorer les formes, mais ce n'est que récemment que le recours à l'expérimentation est devenu systématique. En particulier, un travail important a été fait par I'I. F. P. et le CNEXO pour analyser le comportement et la cinématique des ancres, comportant deux volets : la reconnaissance et l'influence des paramètres permettant l'ouverture, la pénétration, la stabilité des ancres d'une part et l'étude comparative des modèles existants d'autre part (Puech, Meunier et Paillard, 1978).

A partir de 1970, pour la recherche pétrolière en haute mer, la nécessité d'immobiliser à poste de travail fixe des engins flottants pour l'exploitation ou le service des installations, a créé de nouveaux besoins et on a vu apparaître une nouvelle génération d'ancres de très haute capacité (Flipper Delta, Stevin, Bruce, etc.).

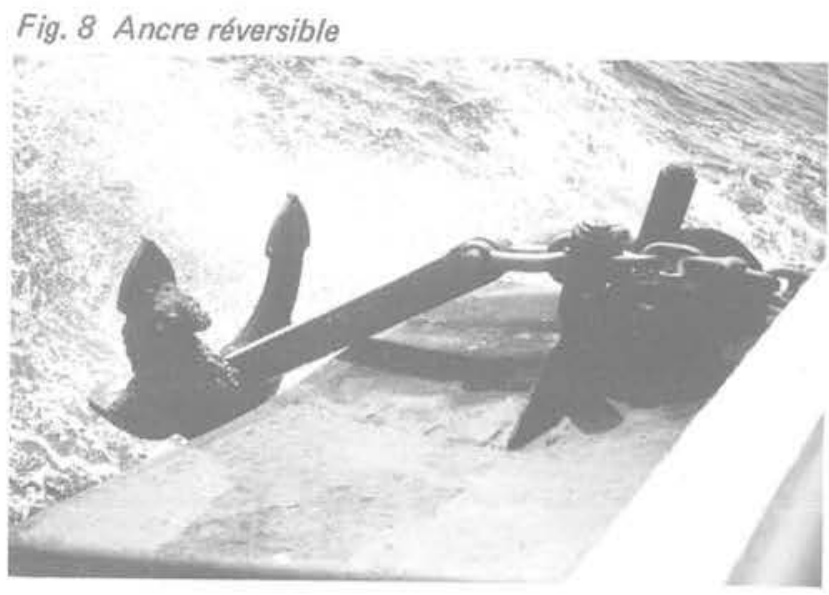

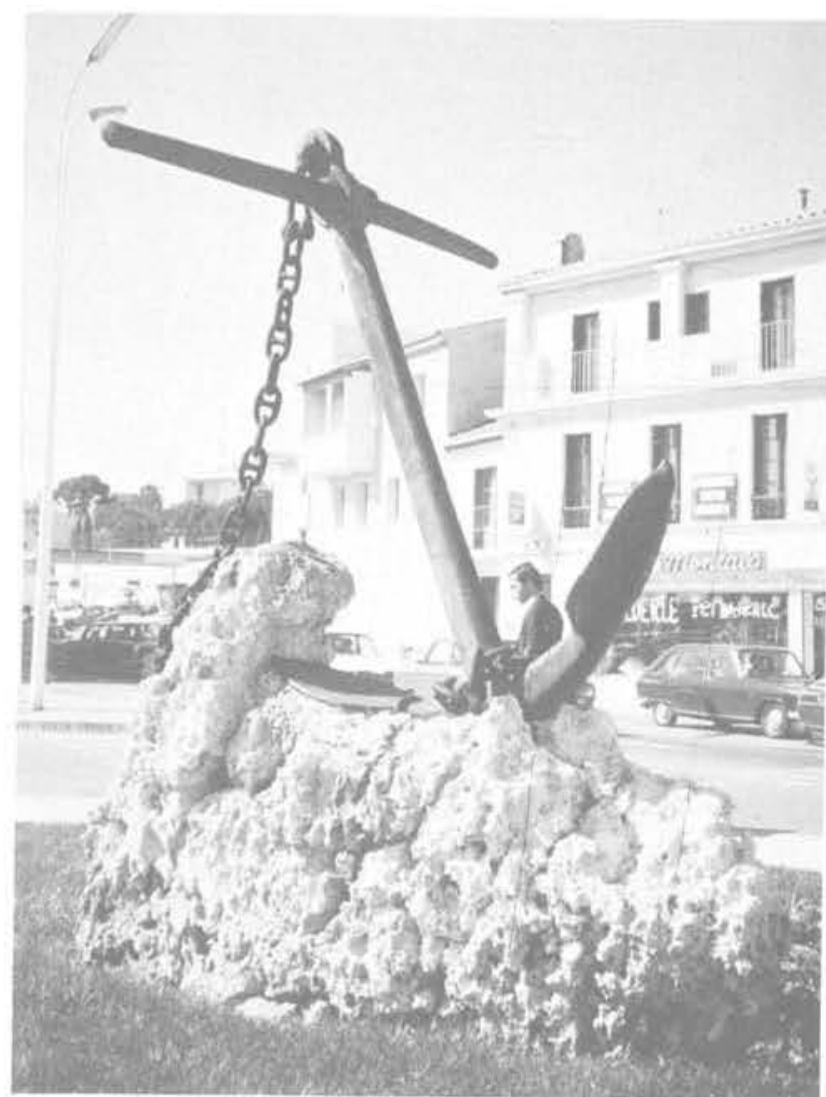

Fig. 5 Ancre à jas

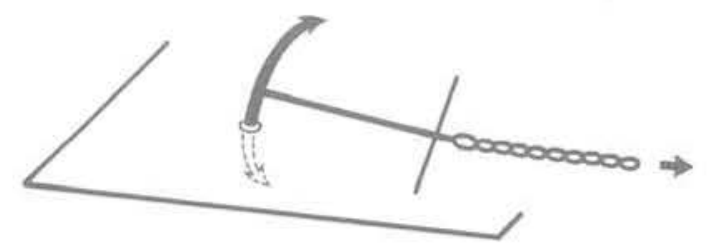

Fig. 6 Positionnement de l'ancre à jas
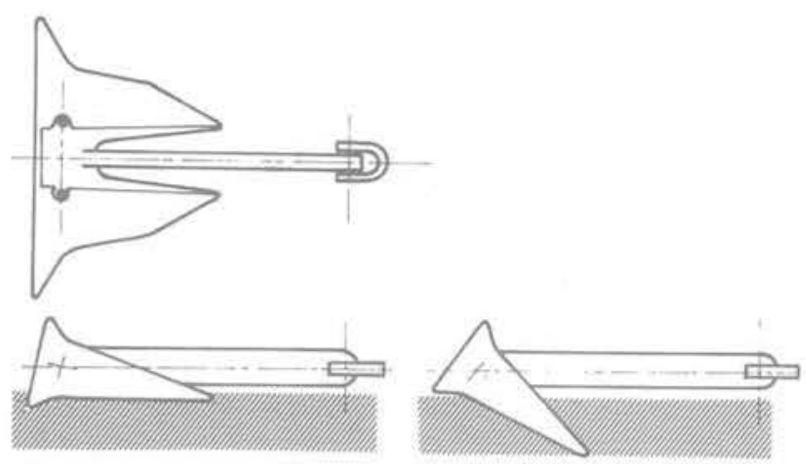

Fig. 7 Ancres à pattes articulées

Fig. 9 Ancre articulée

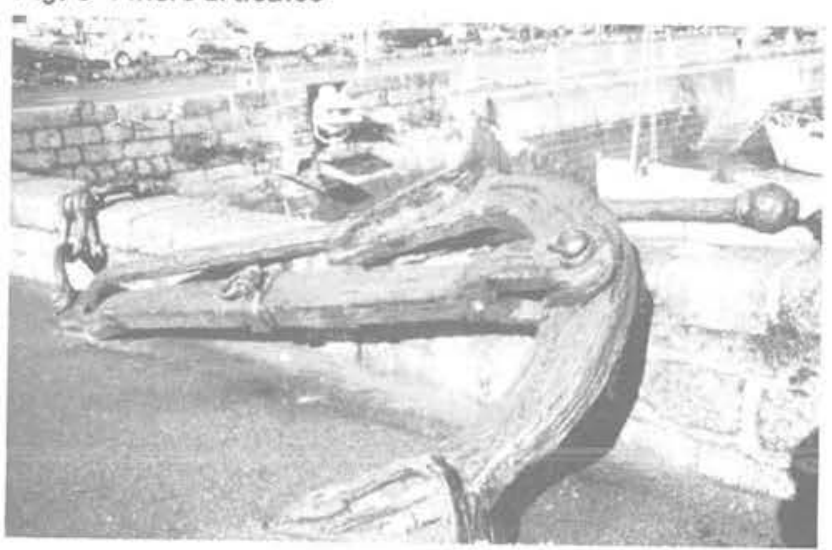


Ce sont de véritables monstres et on a dû abandonner certaines facilités d'emploi des ancres classiques, en particulier on peut avoir besoin de navire auxiliaire pour les mettre en place. Pour ces ancres, comme pour les matériels antérieurs, on retrouve la notion d'efficacité considérée comme le rapport de la force de traction horizontale limite au poids de l'ancre. On verra plus loin que cette notion est ambiguë. Disons cependant, pour l'instant, que pour les forts tonnages le rapport varie de 6 à 10 selon les modèles et selon les fonds. C'est-à-dire que pour des ancrages de 1,5 à $2 \mathrm{MN}(150$ à $200 \mathrm{t}$.), le poids des ancres peut atteindre 0,25 à $0,3 \mathrm{MN}(25$ à $30 \mathrm{t}$.) : à l'évidence, ce sont des engins difficiles à manipuler. Or, les besoins de l'industrie du pétrole pour l'ancrage de longue durée (plusieurs mois) des plates-formes en mer, en particulier des semi-submersibles, vont croissant et ont fait apparaître la nécessité de points d'ancrage plus résistants encore, de l'ordre de 2 à 5 MN (200 à 500 t.). L'extrapolation des engins actuels conduit à des objets de 0,5 à $0,8 \mathrm{MN}(50$ à $80 \mathrm{t}$ ), dont la manipulation devient de plus en plus malaisée; il est alors nécessaire pour ces * fondations " d'une dimension un peu inhabituelle d'accepter de nouvelles servitudes, en particulier de les placer sur le fond et éventuellement de les y ancrer, comme cela se fait d'ailleurs pour les pieux, et d'abandonner les notions de matériels réversibles et de matériels polyvalents.

Bien qu'avec des forces d'ancrage beaucoup plus petites, la navigation de plaisance, dont le développement a été considérable dans les dix dernières années, a rencontré des problèmes d'ancres qui lui sont tout à fait spécifiques. Elle a contribué à sa mesure à un certain effort de recherche et l'on trouve actuellement des références expérimentales sur la tenue des modèles d'ancres classiques à jas ou des grappins, ou des ancres réversibles (Britany, Fob, Fob HP, Salle, etc.), ainsi que d'autres modèles, ancres à soc (CQR) ou ancres en forme de pelle trilobée qui se placent d'elles-mêmes dans le sol (Bruce) et qui sont des "retombées" des ancres modernes de grandes dimensions.

\section{Nouvelles ancres}

Quel que soit le principe utilisé, il est bien évident que pour obtenir un bon ancrage dans le sol il faut s'y enfoncer très profondément. Nous avons donc cherché des modèles pouvant pénétrer dans les fonds les plus divers et après un certain nombre de tentatives plusieurs formes ont été retenues, qui sont soit apparentées à des formes classiques, mais d'assez loin, et progressivement optimisées, soit d'un principe entièrement nouveau et qui correspond en quelque sorte à un planeur souterrain. Nous allons l'examiner maintenant.

Imaginons un cerf-volant: il s'élève dans le ciel tant que l'angle entre la ligne d'accrochage et le plan du cerf-volant est inférieur à un angle droit (fig. 10). II s'arrête de monter lorsque cet angle atteint $90^{\circ}$ et il acquiert alors une certaine instabilité lui permettant un mouvement perpendiculairement au plan vertical de la ligne et d'ailleurs il peut s'abattre jusqu'au sol en paraissant circuler sur une surface de révolution. On remédie à cette instabilité en augmentant la traînée, par exemple au moyen d'une queue; la ficelle de la ligne d'accrochage n'est pas rectiligne puisqu'elle est poussée par le vent; elle se redresse plus sa cote augmente.

Prenons maintenant la figure symétrique (fig. 11). Le

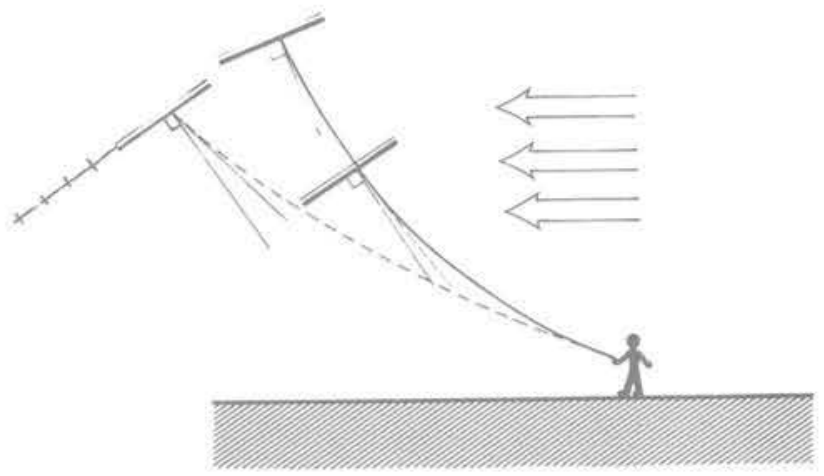

Fig. 10 Principe du cerf-volant
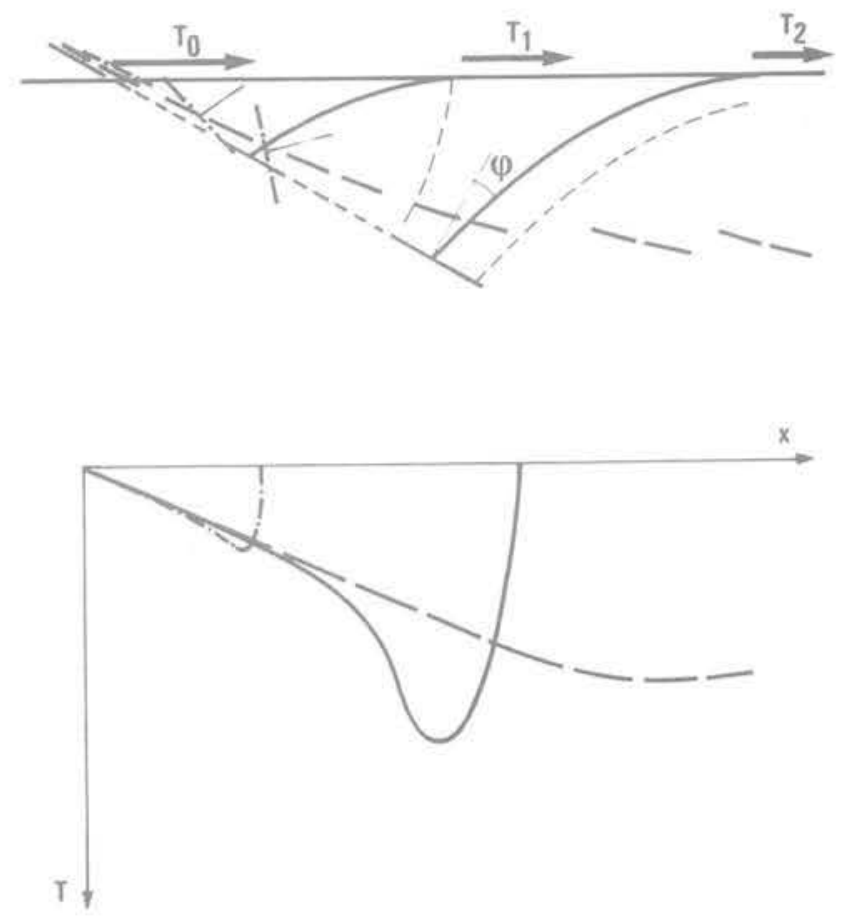

Fig. 11 Mécanisme d'enfouissement de la nouvelle ancre

cerf-volant est une plaque qui s'enfonce dans le sol. La ligne d'accrochage poussée par le « vent » est un câble suffisamment fin pour que sa traînée soit la plus petite possible et qu'il fende la terre comme un fil à couper le beurre.

La plaque s'enfonce au fur et à mesure de l'augmentation de la traction et son mouvement s'arrête lorsque l'angle de la ligne et de la normale à la plaque atteint l'angle de frottement du sol sur la plaque. Lorsqu'il s'agit d'un sable et d'une plaque métallique, cet angle est de l'ordre de 25 à $30^{\circ}$. S'il s'agit d'une argile, la tangente à la ligne en son point d'amarrage sur la plaque peut atteindre la normale à la plaque, avec l'apparition d'une instabilité qu'on pourra combattre avec une queue, comme pour le cerf-volant, ou plus simplement en donnant à la plaque une section transversale en W, car le sol est un matériau plastique, c'est-à-dire présentant un seuil de résistance et il suffit de créer un obstacle au mouvement latéral pour donner une très grande rigidité à la trajectoire de la plaque.

Si on augmente l'effort lorsque l'enfoncement est arrivé à sa plus grande valeur et que la plaque est arrêtée, on mobilise progressivement la butée audessus de la plaque puis on arrache la plaque 

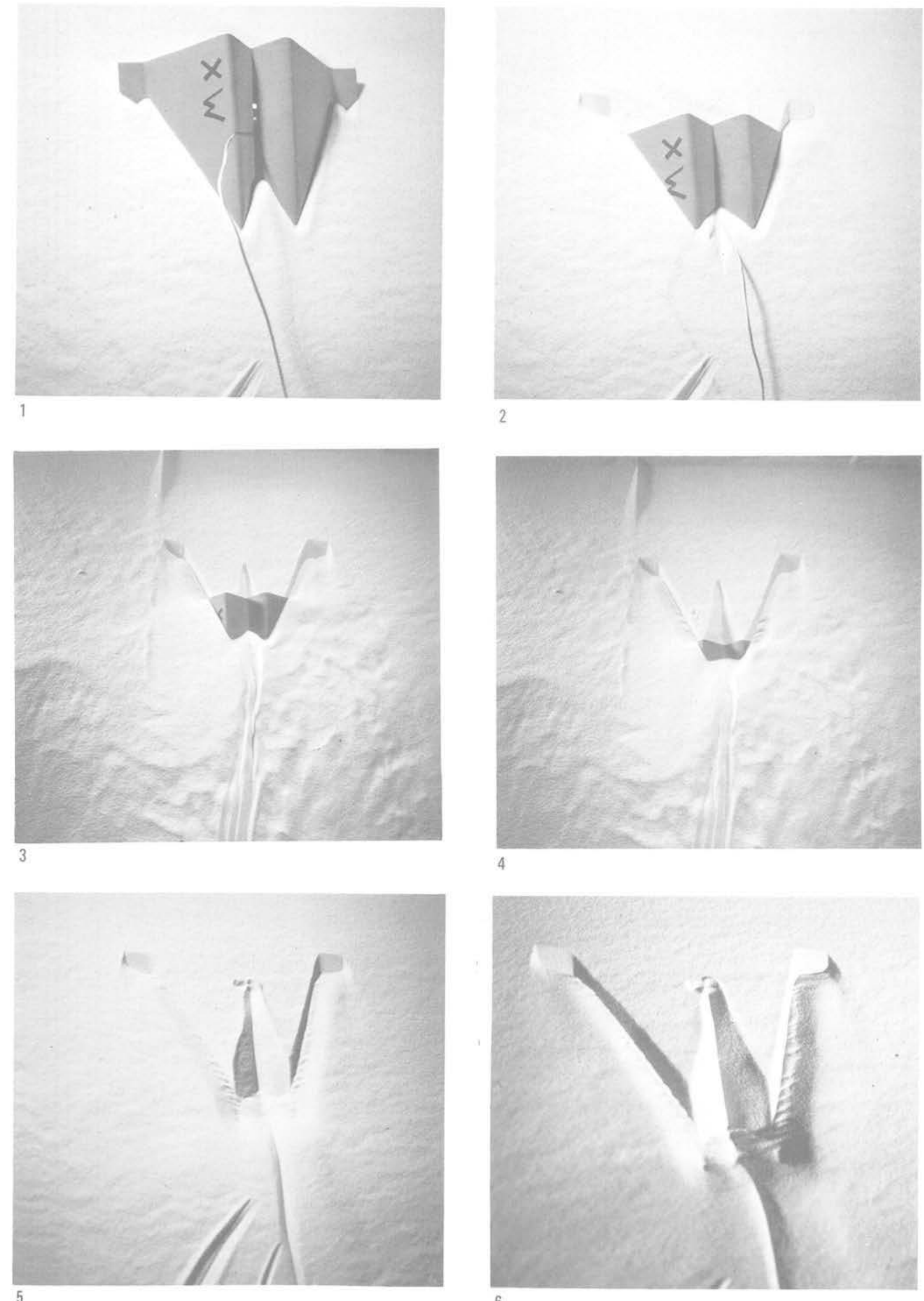

Fig. 12 Pénétration d'une ancre $X W$ dans du sable sec 
d'ancrage ainsi qu'un cône de terre, c'est-à-dire avec un effet tridimensionnel qui peut donner une résistance considérable. On peut donc s'attendre à ce que la forme de la courbe de l'effort horizontal en fonction du déplacement ait l'allure indiquée sur la figure 11 en trait plein.

L'expérience est cependant indispensable pour voir si, pour une ligne accrochée trop à l'avant de la plaque, la trajectoire de l'ancre s'infléchit vers le haut ou se décale horizontalement par translation, ce qui ne manquerait pas de donner à la courbe effortdéplacement l'allure de la courbe en tireté de la figure 11. Mais surtout, il est à craindre que l'ancre bascule au cours de son introduction dans le sable, ou même un peu après son enfoncement complet, et qu'elle se renverse sous l'effet du couple de la force horizontale et de la résistance du sol, auquel s'oppose seulement le moment du poids propre de l'ancre et qui peut être insuffisant. On obtiendrait alors la courbe en trait mixte de la figure 11 associée à une trajectoire très courte. En somme, si on associe d̀ l'ancre un axe ox dans la direction du mouvement et un axe oy normal à la plaque, il faut s'assurer de l'équilibre suivant les abscisses (glissement), suivant les ordonnées (arrachage) et de l'équilibre des moments (renversement); l'expérience montre que cette dernière condition est primordiale au cours des premiers instants de la pénétration et elle est capitale puisque le renversement de l'ancre empêche toute possibilité de pénétration ultérieure. La figure 12 montre une séquence photographique de la pénétration d'une maquette dans du sable sec.

Cette forme d'ancre n'est pas parmi les meilleures. Essayée en mer (fig. 13), elle n'a pas donné toute satisfaction, en particulier parce que les modèles réels s'éloignent du schéma de principe présenté ci-dessus, d'une part par la résistance à la pénétration du bord de la plaque qui n'est pas négligeable et qu'on a intérêt à minimiser, par exemple en lui donnant la forme d'un dièdre très aigu, d'autre part par la traînée de la ligne d'ancrage qui s'appuie et bouscule le massif de butée devant la plaque.

De toutes façons, il fallait avoir recours à l'expérimentation pour choisir les formes les mieux adaptées; mais avec des forces aussi grandes que celles qui sont envisagées dans l'industrie pétrolière, la mise au point de différents modèles en vraie grandeur est pratiquement impossible. On est alors obligé d'utiliser des modèles réduits, et cela d'autant plus que dans une configuration tridimensionnelle le calcul en plasticité n'est pas très réaliste, en particulier lorsque $\varphi \neq 0$.

\section{Essais en similitude}

Rappelons les principes de la similitude en Mécanique des Solides utiles à cette étude particulière de Mécanique des Sols (Mandel, 1962).

II faut s'assurer que le modèle et le prototype sont mécaniquement semblables et pour cela, deux groupes d'équations doivent être vérifiés.

II y a d'abord les équations générales de l'équilibre statique. Si l'on note ù l'échelle d'une certaine grandeur u, c'est-à-dire le rapport de la mesure de cette grandeur dans le modèle à sa mesure dans le prototype, les conditions d'homogénéité des équations donnent, avec les notations évidentes :

$\dot{\sigma} \dot{\ell}^{-1}=\ddot{\rho} \dot{g}$.

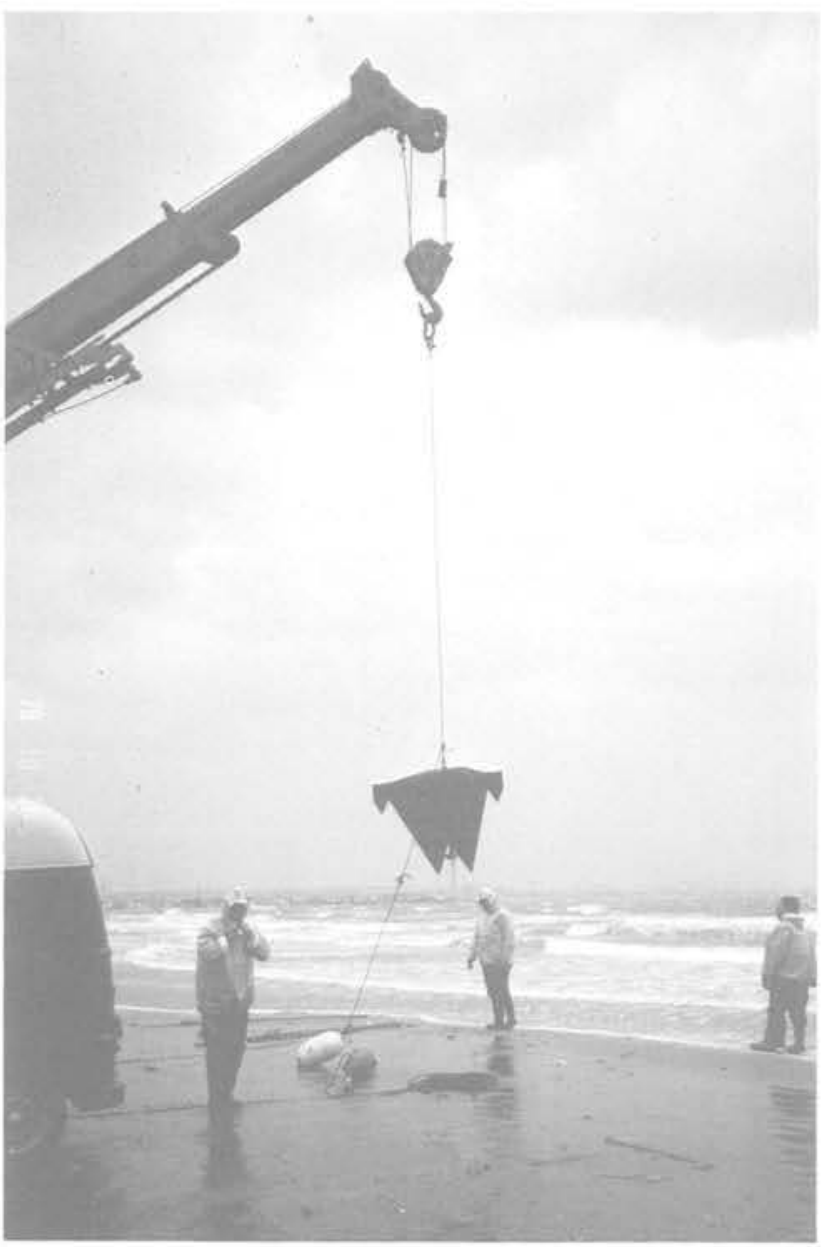

Fig. 13 Ancre XW de $1300 \mathrm{~N}$ sur la plage de Fos-sur-Mer

En conditions ordinaires d'expérimentation, on a $\dot{g}=1$ et $j=1$ (ce qui signifie, dans notre cas particulier, que l'expérimentation est faite sous l'eau avec les mêmes sols et les mêmes métaux pour avoir les mêmes poids volumiques déjaugés). Ceci entraîne :

$\dot{\sigma}=\dot{\ell}$,

et pour les poids et les forces :

$\dot{\mathrm{F}}=\dot{\sigma} \cdot \dot{\mathrm{S}}=(\dot{\ell})^{3}$.

Cette dernière relation est particulièrement intéressante lorsqu'on cherche à représenter de très grandes forces.

II faut vérifier ensuite les équations rhéologiques. Pour l'étude des ancres, on ne s'intéresse pas aux petites déformations: on peut donc négliger les relations tirées de l'élasticité. Par contre, il faut s'assurer de la similitude des critères de rupture et ceci entraîne :

$\dot{\varphi}=1$

(conserver l'angle de frottement interne, c'est-à-dire en fait conserver le même sable à la même densité).

et :

$\dot{\mathrm{c}}=\dot{\alpha}=\dot{\ell}$.

Enfin, il faut respecter le développement - et la dissipation - de la pression interstitielle.

La durée de la dissipation de cette pression est donnée par :

$t=\frac{T a_{v} \ell^{2}}{k\left(1+e_{0}\right)}$

d'où la relation de similitude :

$\dot{t}=\dot{\ell}^{2} \dot{k}^{-1}$, 
et elle donne une condition entre la vitesse de déplacement de l'ancre et la perméabilité :

$\dot{v}=\ddot{\ell} \grave{t}^{-1}=\hat{\ell}^{-1} \cdot \dot{k}$.

Lorsque toutes les conditions sont vérifiées, I'expérimentation sur petits modèles doit permettre une prévision acceptable pour les grands prototypes. Qu'en est-il en pratique? En laboratoire et dans des sables secs, la théorie est assez bien respectée pour des forces allant de $50 \mathrm{~N}$ à $2000 \mathrm{~N}$ (ce qui ne fait d'ailleurs que 3,5 pour l'échelle des longueurs). Pour les modèles plus grands, cela va moins bien et l'on constate que le rapport $\frac{T}{P}$ varie en fonction de $\mathrm{P}$ dont il est une fonction décroissante.

La figure 14 représente, en coordonnées logarithmiques pour des ancres de poids $\mathrm{P}$, la valeur des forces horizontales T nécessaires pour obtenir la qualification du Bureau Veritas (1975). Nous y avons porté d'autres données relatives à une ancre classique à jas et à une bonne ancre réversible de plaisance, ainsi que les résultats de nos essais sur un de nos modèles dans divers sables. Dans cette présentation les courbes d'égale efficacité $\frac{T}{\mathrm{P}}$ sont des droites inclinées à $45^{\circ}$. On voit que l'efficacité des ancres réversibles varie de 70 à 6 lorsque le poids passe de $120 \mathrm{~N}(12 \mathrm{~kg})$ à $350 \mathrm{KN}$ $(35 \mathrm{t})$. Ceci montre à l'évidence que la similitude n'est pas très bonne et qu'il existe un effet d'échelle. C'est d'autant plus désagréable que le phénomène du renversement initial est affecté par cet effet et que la pénétration est plus difficile pour les grands modèles que pour les petits : le réglage exact de la position du point d'accrochage est donc difficile à transposer des petits modèles aux grands, surtout lorsqu'on ne dispose que du poids propre pour équilibrer le moment de renversement. Or, on se rend compte intuitivement qu'il est souhaitable d'accrocher la plaque le plus en arrière possible; en effet, si elle est tirée par un point situé trop à l'avant, elle s'arrachera plus facilement de terre du fait de la distribution non uniforme des contraintes normales à la plaque.

Mais, on peut aussi envisager la mise en place d'un dispositif stabilisateur destiné à augmenter le moment résistant au renversement initial. C'est ce qui a été fait avec le modèle de la figure 15, qui présente une séquence photographique de la pénétration dans un sable sec. Les figures 16,17 et 18 représentent un prototype sur une plage, sa mise en mer et le début de la pénétration. La figure 19 représente l'extraction du modèle le plus performant qui a été construit jusqu'à présent. On voit sur la figure 14 que pour une même force horizontale $T$ le poids de ces modèles est, en moyenne, six fois plus petit que celui des ancres réversibles.

La similitude dans l'argile semble donner par contre des résultats beaucoup plus conformes à la théorie. Ainsi, nous avons obtenu la pénétration en laboratoire d'une maquette de $12 \mathrm{~cm}$ de long dans une argile très molle de $2,5 \mathrm{kPa}\left(25 \mathrm{~g} / \mathrm{cm}^{2}\right)$ de cohésion. Ce modèle a été reproduit ensuite à l'échelle 10 , ayant ainsi une longueur de $1,20 \mathrm{~m}$ (fig. 20 et 21 ), et il a réussi à pénétrer dans une argile dont la cohésion était comprise entre 20 et $30 \mathrm{kPa}\left(200\right.$ à $\left.300 \mathrm{~g} / \mathrm{cm}^{2}\right)$. II s'est enfoui ensuite jusqu'à une profondeur de $3,4 \mathrm{~m}$ en mobilisant une force de $27 \mathrm{kN}(2,7 \mathrm{t})$ en accord avec le résultat prévu par l'essai sur modèle en laboratoire, c'est-à-dire avec une échelle des forces de $10^{3}$ comme le montre de façon évidente la figure 22. La similitude pour les milieux cohérents doit évidemment tenir

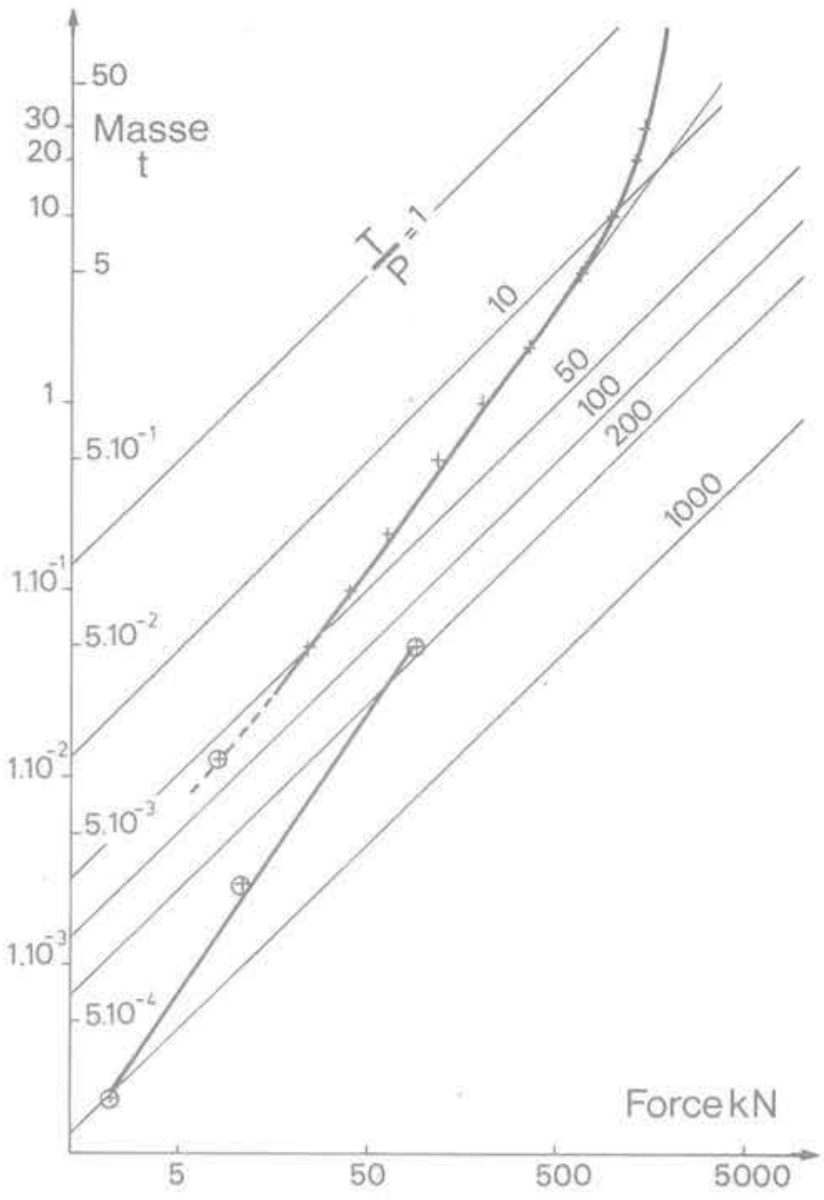

Fig. 14 Efficacité des ancres marines (qualification du bureau Véritas, 1975)

compte de la pression atmosphérique et de la présence de la nappe.

La similitude paraît moins satisfaisante pour les sables que pour les argiles. On remarquera cependant la régularité des courbes de la figure 14, pour en déduire qu'il n'est pas essentiel de connaître l'origine de l'effet d'échelle pour utiliser la similitude comme moyen d'extrapolation des modèles. Sur la base des résultats actuels, il nous paraît raisonnable de prévoir des sauts de 5 sur l'échelle des forces, ce qui représente des modèles 1,7 fois plus grands pour l'échelle des longueurs. C'est le choix qui a été fait pour le prochain prototype.

On trouvera, ci-dessous, les principaux résultats obtenus avec les maquettes du meilleur des modèles essayés :

- Modèle réduit au L.M.S. :

$\ell$ Longueur des modèles

$15 \mathrm{~cm}$

$\mathrm{L}$ Longueur de la cuve $\quad: 1,50 \mathrm{~m}$

F Force maximale $\quad: 2 \mathrm{kN}$

- Modèle réduit à I'IFP:

$\ell$ Longueur des modèles $\quad: 60 \mathrm{~cm}$

$\mathrm{L}$ Longueur de la cuve $\quad: 5 \mathrm{~m}$

F Force maximale $\quad: 17 \mathrm{kN}$

- Modèle sur une plage :

$\ell$ Longueur des modèles $: 1 \mathrm{~m}$ (poids $500 \mathrm{~N}$ )

F Force maximale $\quad: 86 \mathrm{kN}$ 

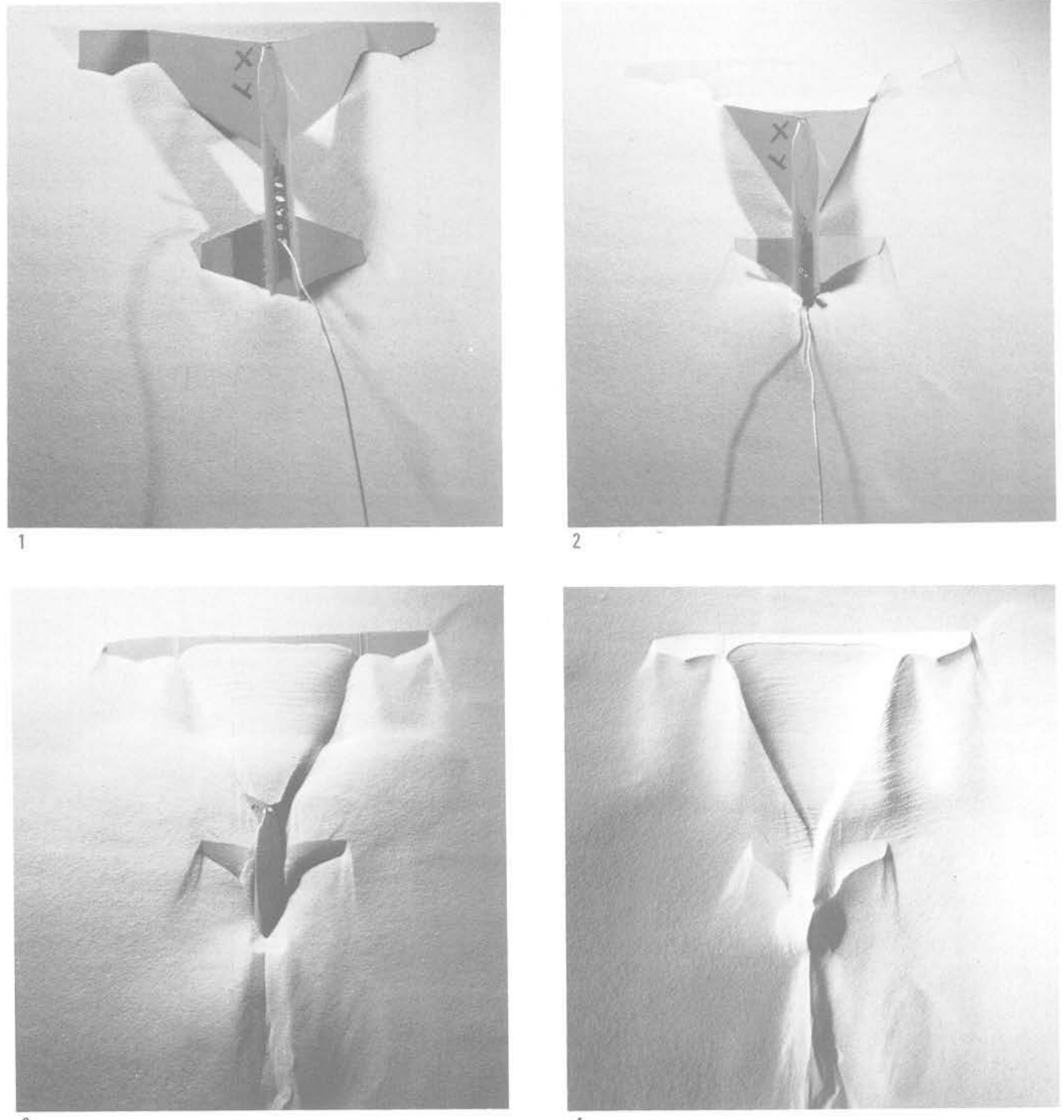

Fig. 15 Pénétration de l'ancre $X Y$ dans un sable sec

Fig. 16 Prototypes d'ancres de $1000 \mathrm{~N}$
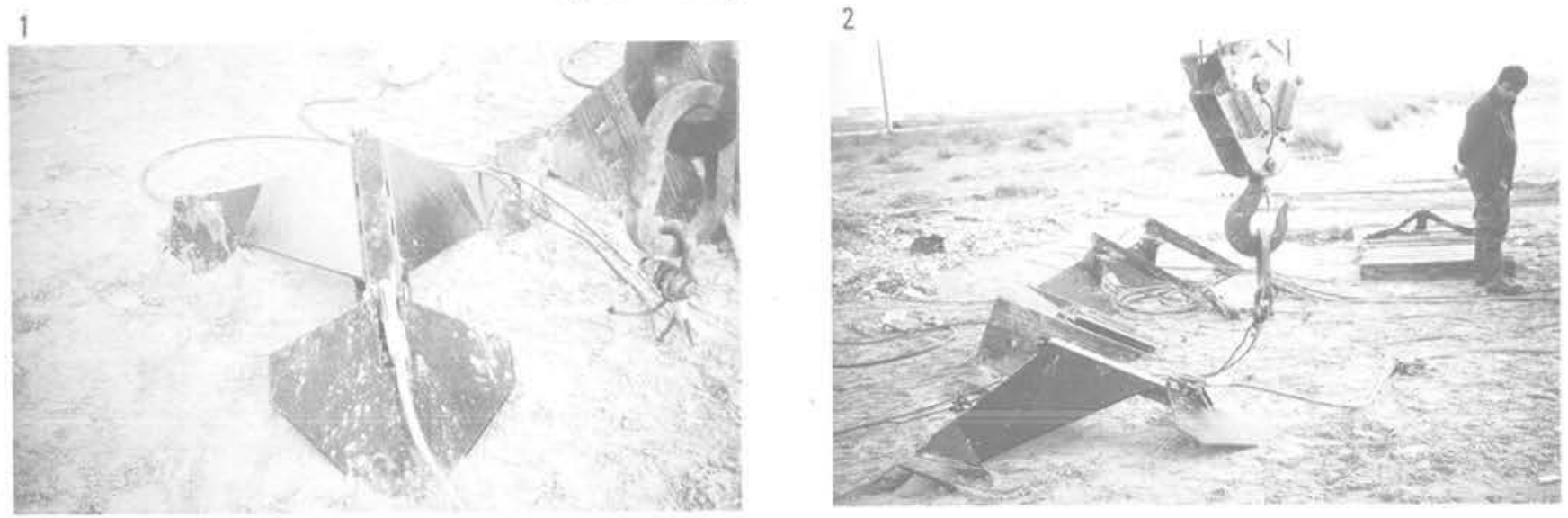

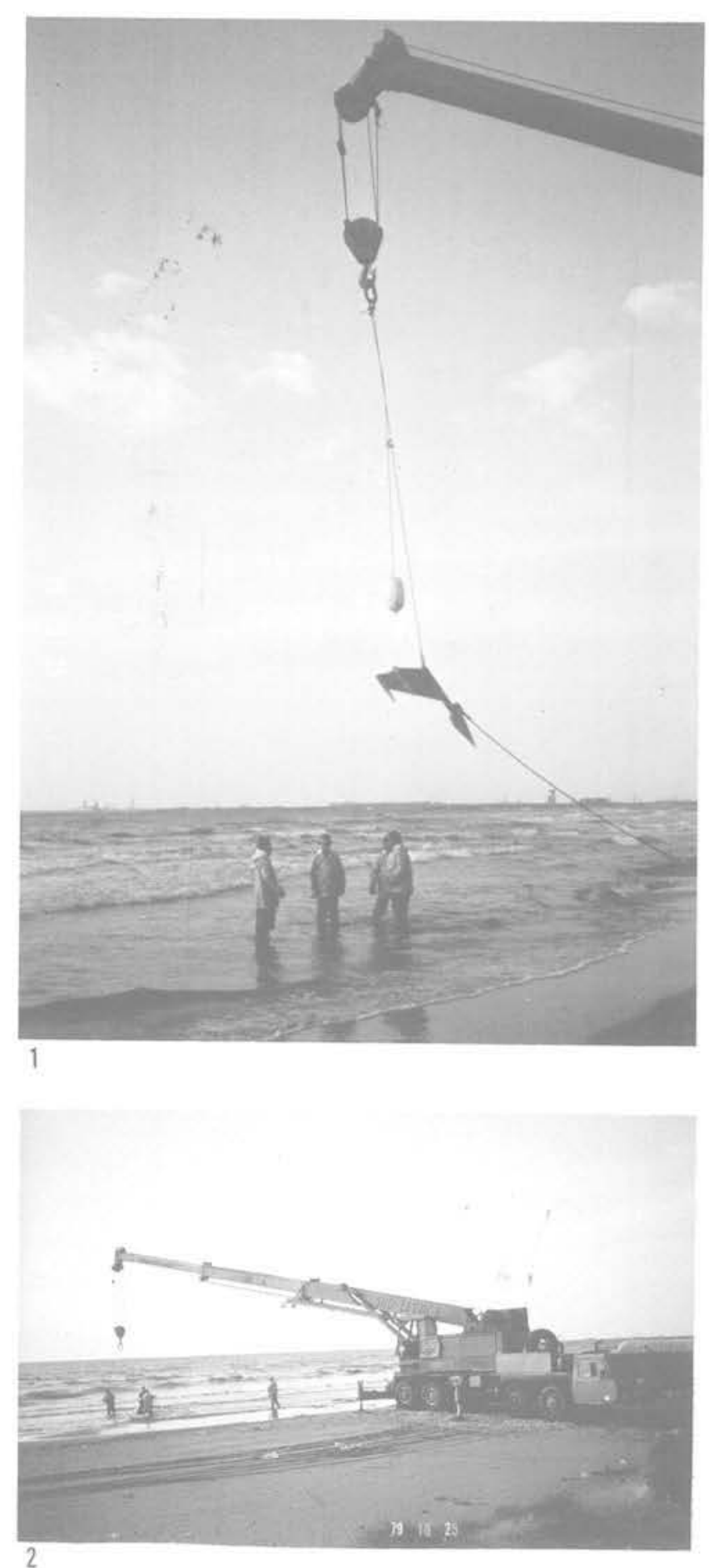

Fig. 17 Essais d'ancre à Fos-sur-Mer

Fig. 18 Prototype XY de $500 \mathrm{~N}$

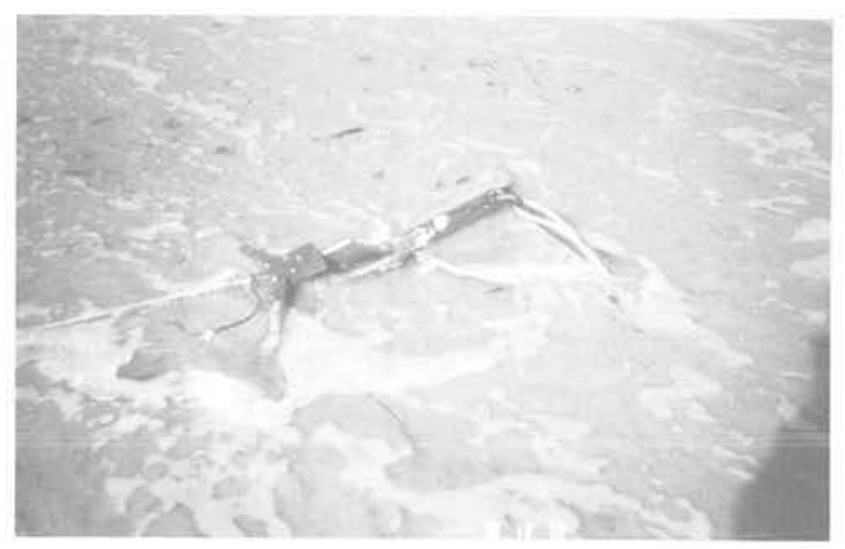

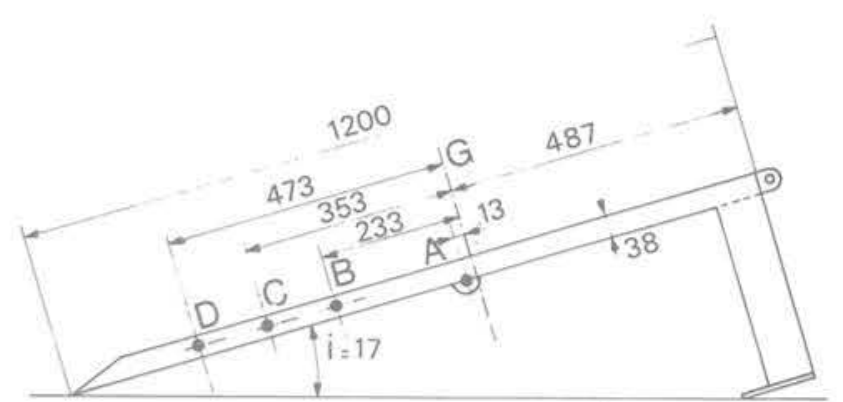

cotes en mm

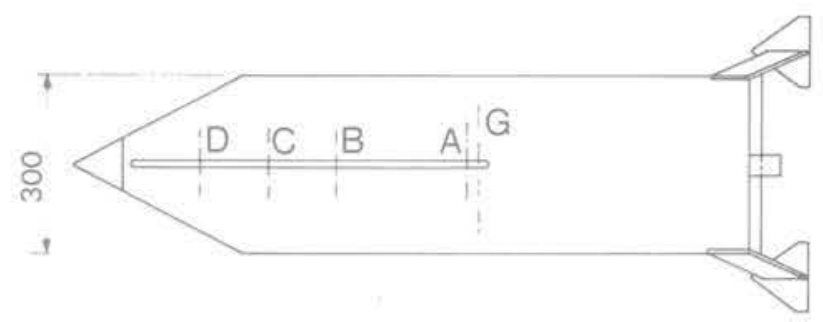

Fig. 20 Scherma d'une ancre-plaque

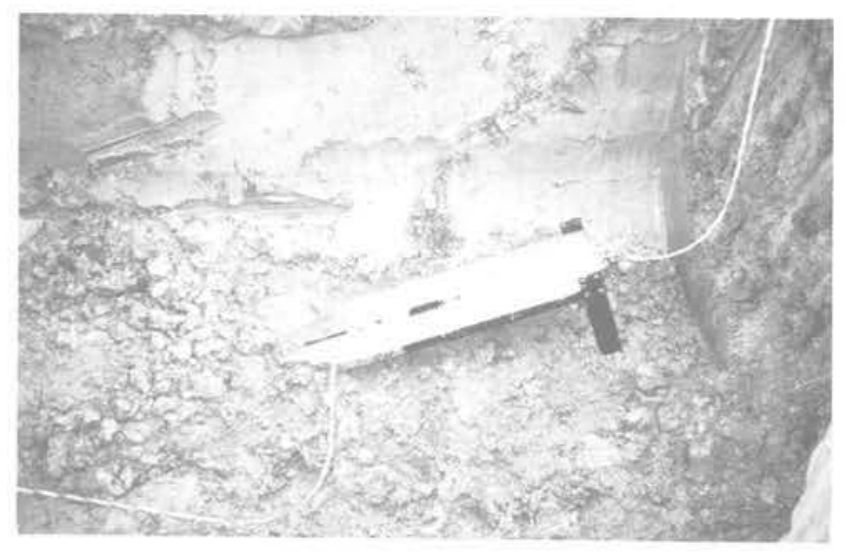

Fig. 21 Essai d'ancre-plaque dans I'argile de Cran

Fig. 19 Extraction du prototype XY de $500 \mathrm{~N}$

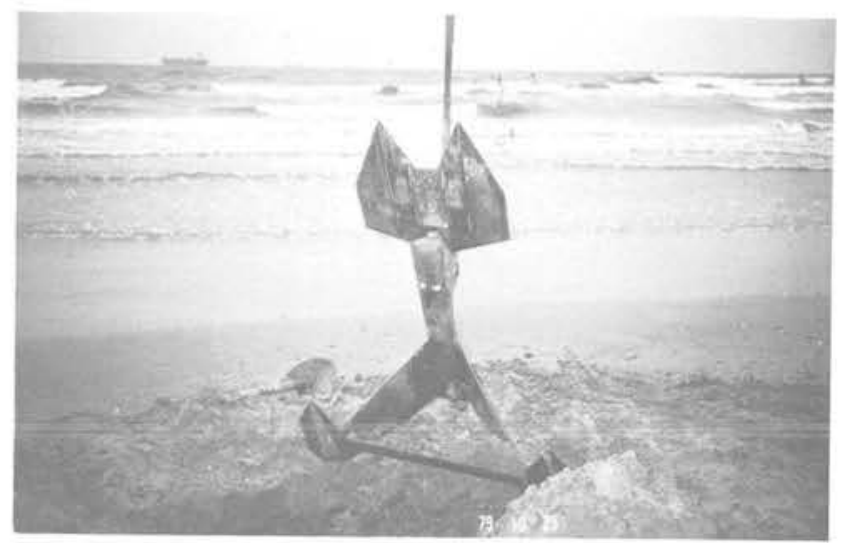




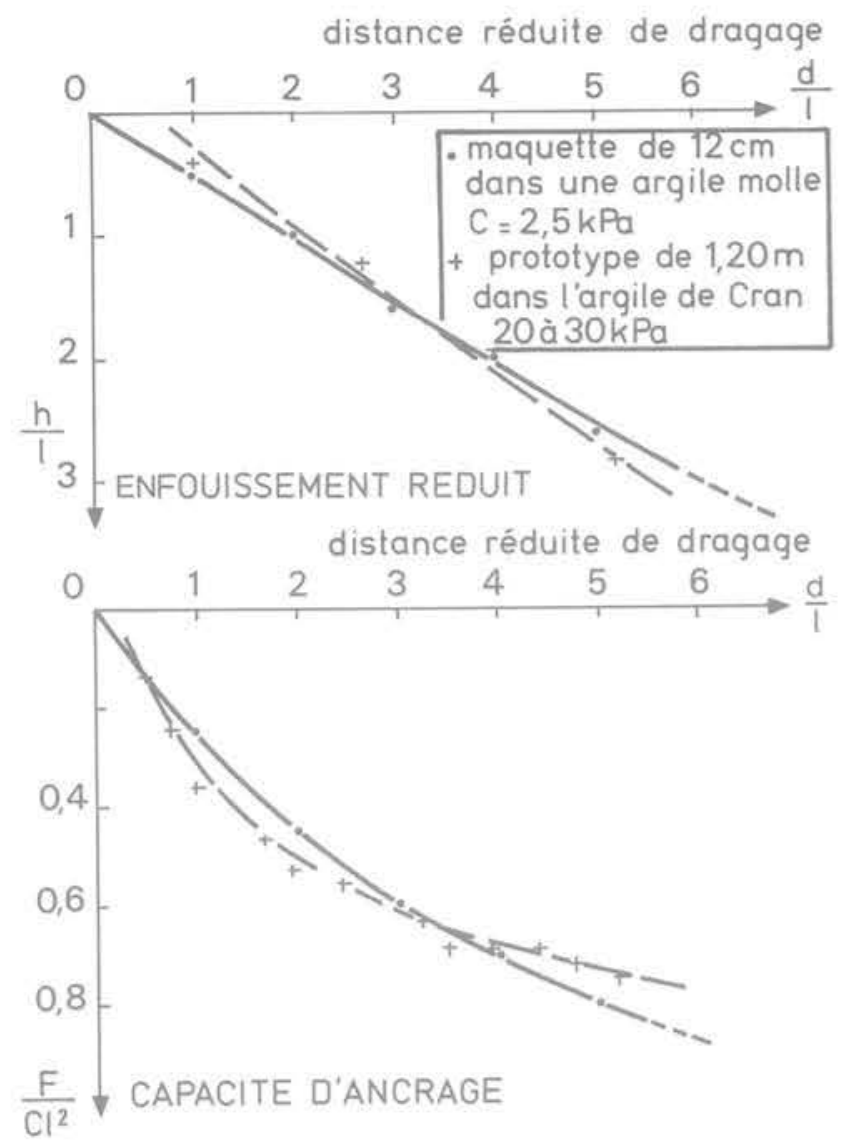

Fig. 22 Performances comparées de la maquette de $12 \mathrm{~cm}$ et du prototype de 1,20 $\mathrm{m}$ de longueur

Fig. 23 Déviation de la ligne d'ancrage

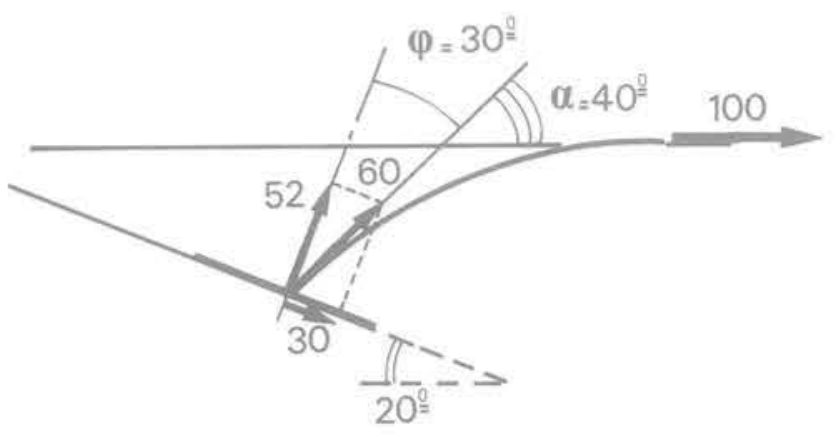

$\alpha=\frac{\pi}{2}-20^{\circ}-\varphi=40^{\circ}$

$-\mathrm{f} \boldsymbol{\alpha}$

e $=0,6$

\section{La ligne d'ancrage}

La ligne d'ancrage transmet au massif des efforts considérables. Sur la figure 23 , la plaque est arrêtée par le frottement alors qu'elle pénétrait dans le sable sous une incidence $\mathrm{i}=20^{\circ}$; on voit que la déviation maximale $\alpha$ de la ligne d'ancrage est :

$\alpha=\frac{\pi}{2}-i-\varphi=40^{\circ}$.

Or, la ligne frotte sur le massif de sable; le rapport de la force appliquée à la plaque à la traction horizontale exercée hors du sol, vaut donc $e^{-\alpha \operatorname{tg} \varphi} \# 0,6$. Ainsi, à l'équilibre limite $40 \%$ environ de l'effort d'ancrage est transmis au massif de sable par la ligne d'ancrage. On voit donc l'intérêt d'utiliser un câble le plus fin possible de façon à pouvoir tendre la trajectoire de la ligne d'ancrage dans le sol : son inclinaison par rapport à la normale à la plaque deviendrait alors supérieure à $\varphi$ et le mouvement de glissement pourrait reprendre; la plaque s'arrêterait alors un peu plus bas, lorsque la courbure de la ligne permettra de retrouver un angle $\alpha=40^{\circ}$. Ainsi, plus la ligne est fine plus l'ancre s'enfonce profondément, et plus la tension de la ligne est grande. Cet effet est particulièrement net sur les figures 24 et 25 où des modèles de $30 \mathrm{~cm}$ et de $45 \mathrm{~cm}$ ont été essayés avec des chaînes de différentes grosseurs et avec un câble fin d'acier. Les profondeurs atteintes et les forces limites se rangent dans l'ordre des épaisseurs des lignes d'ancrage.

II faut donc utiliser pour la ligne d'ancrage un matériau ayant une très grande résistance à la traction. Or, il est bien connu que l'emploi des aciers à haute résistance dans l'eau de mer n'est pas conseillé. L'utilisation d'acier ordinaire pour les chaînes, avec des surépaisseurs contre la corrosion, conduit à des lignes d'ancrage extrêmement défavorables, car très épaisses. Nous avons donc été amené à utiliser un câble en kevlar, qui permet des contraintes de traction très importantes. Bien entendu, le kevlar est utilisé uniquement dans le sol; il est prolongé à l'extérieur par une chaîne en acier dont le poids est nécessaire pour ramener la traction de la ligne à l'horizontale. La figure 26 représente un essai avec une maquette de $60 \mathrm{~cm}$ avec destruction de l'ancre, car il y a évidemment aussi pour l'ancre un problème de structure. Enfin, la figure 27 correspond à l'essai d'une maquette de $1 \mathrm{~m}$ dans du sable avec un câble en Kevlar.

\section{Ancrage au sol}

A terre, la cohésion apparente des sols en surface est forte; elle est de l'ordre de 0,1 à 0,2 MPa, par exemple, pour le limon du Plateau; il est donc pratiquement impossible d'espérer y faire entrer une ancre sous l'effet de son propre poids et d'une traction horizontale. II faut souligner d'ailleurs que le rapport des poids volumiques est plus défavorable dans l'air $\left(\frac{7,9}{2,1}=3,7\right)$ que dans l'eau $\left(\frac{6,9}{1,1}=6,2\right)$, ce qui rend l'enfouissement d'une ancre plus aisé au fond de l'eau qu'à terre. La similitude montre que pour pouvoir pénétrer dans le sol, une ancre de ce type devrait avoir près de dix mètres de long, ce qui correspondrait à des efforts énormes, de l'ordre de 20 à $30 \mathrm{MN}(2000$ à $3000 \mathrm{t})$. Le renversement initial apparaît donc de règle pour les petites ancres à terre. II faut alors s'affranchir de ce 

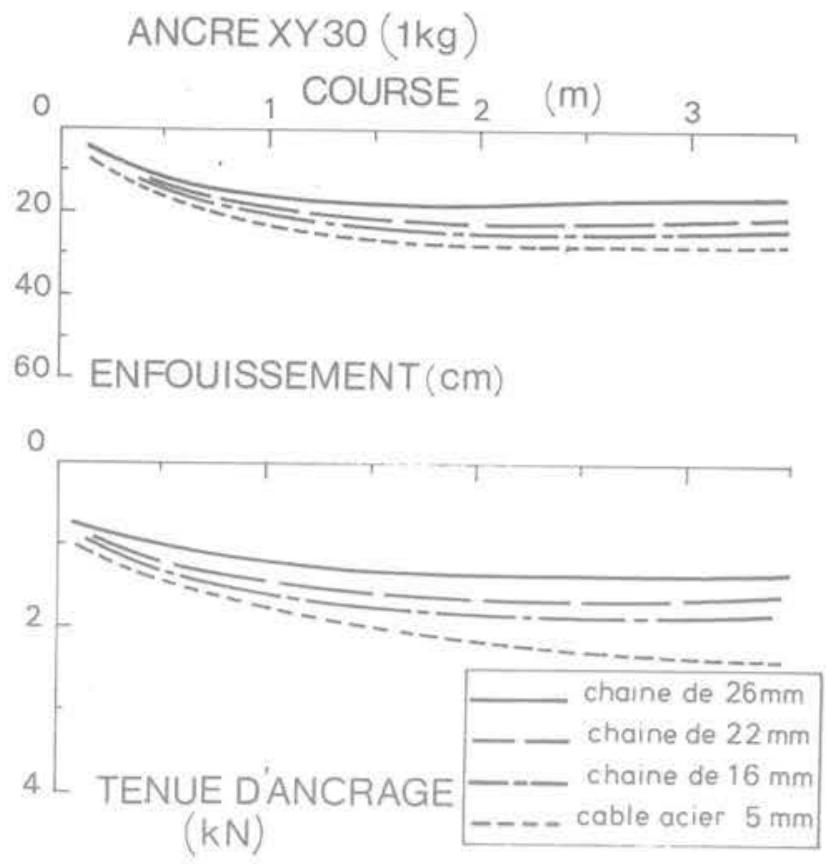

Fig. 24 Influence de la ligne d'ancrage sur la tenue de l'ancre XY 30

Fig. 26 Essais sur deux ancres de $60 \mathrm{~cm}$ de long ANCRE XY60

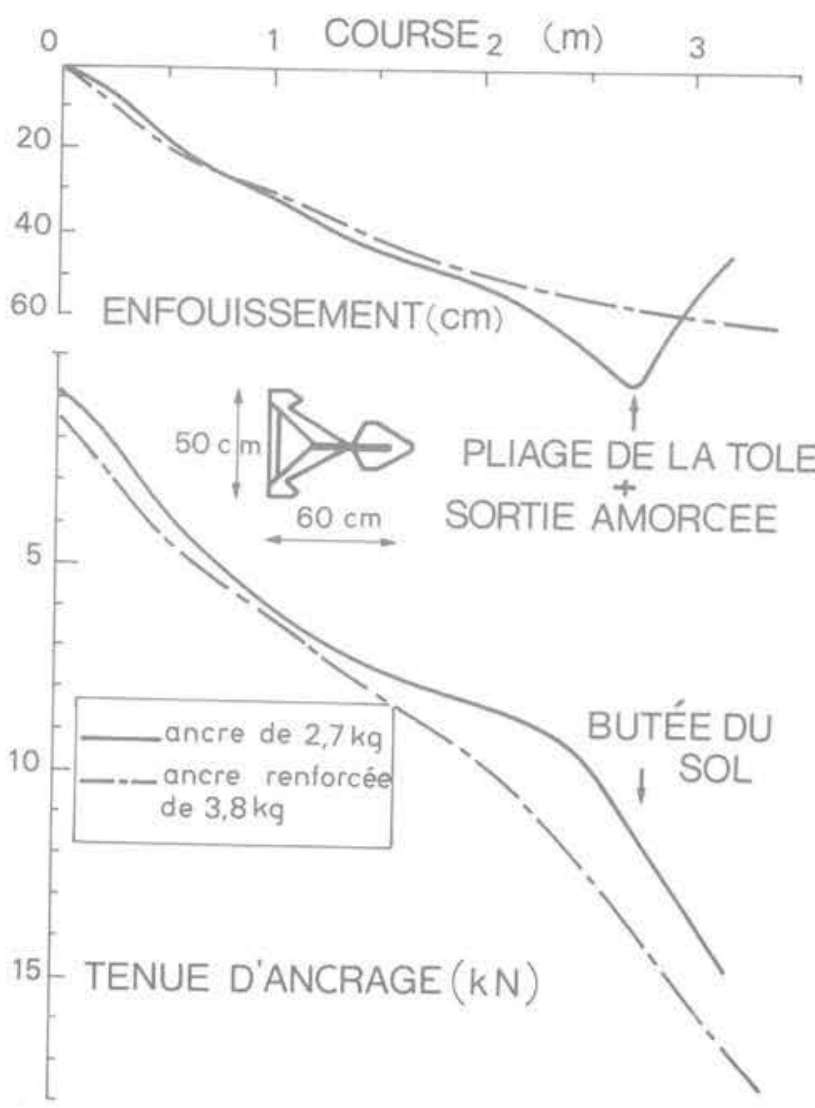

ANCRE XY45 $(1,8 \mathrm{~kg})$
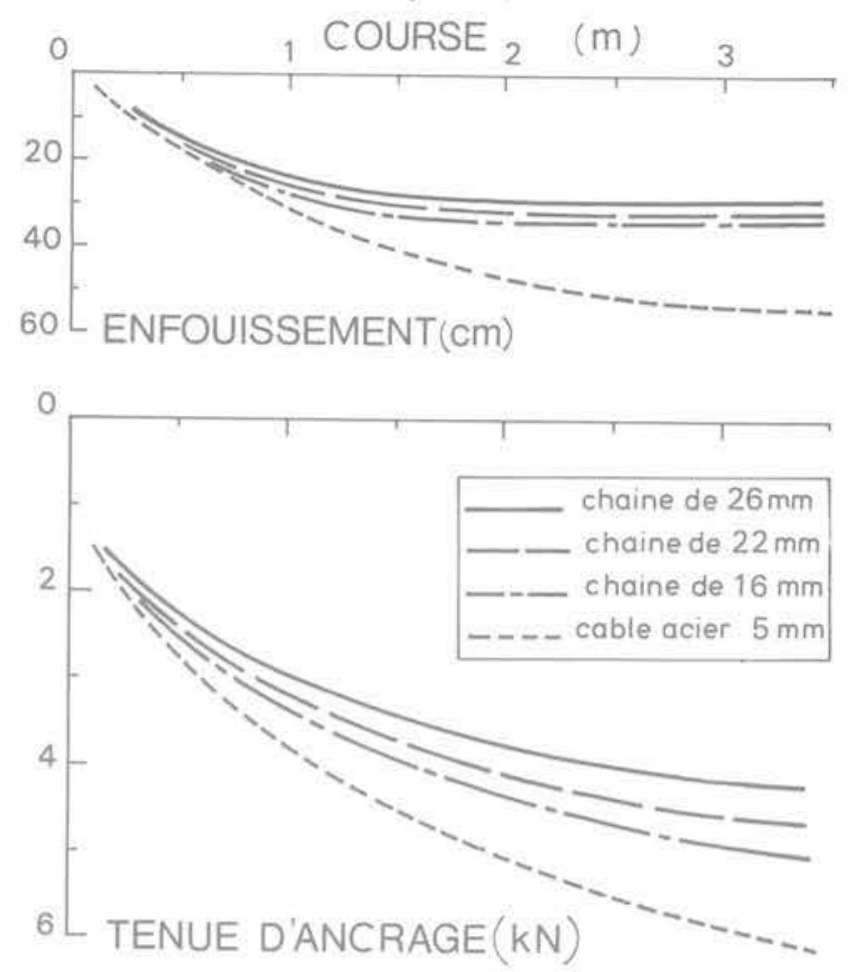

Fig. 25 Influence de la ligne d'ancrage sur la tenue de l'ancre XY 45

Fig. 27 Essai d'une ancre de $1 \mathrm{~m}$ de long avec câble en kevlar dans du sable saturé
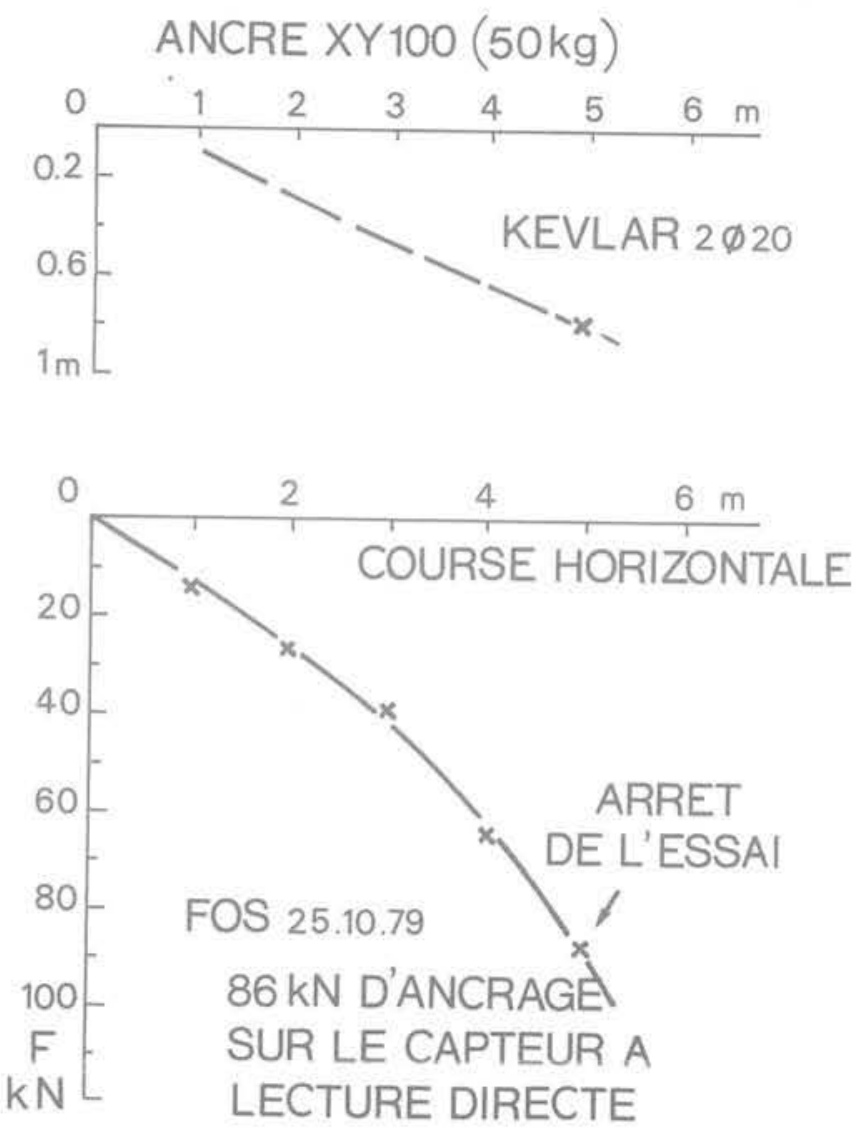
mauvais pas, par exemple en battant la plaque d'ancrage depuis la surface. Du même coup, on peut utiliser une incidence plus forte, car on ne se soucie plus qu'elle soit défavorable au renversement initial, mais elle permet de placer la plaque presque perpendiculairement à la ligne d'ancrage (fig. 28). Cette ligne est battue avec la plaque, puis ramenée vers l'avant en la plaçant dans une fente découpée dans le sol par panneaux successifs par battage d'une pelle. Avec une plaque de $2 \mathrm{dm}^{2}$ enfoncée à $0,60 \mathrm{~m}$ de profondeur, nous avons obtenu d'une façon très répétitive une résistance à l'arrachage de $30 \mathrm{kN}(3 \mathrm{t})$ à comparer à la force de battage qui est à peu près vingt fois plus petite. Ceci montre tout l'avantage qu'on peut obtenir de l'effet tridimensionnel et de l'appui latéral en butée à partir d'un ancrage quasiponctuel. Ces résultats permettent de prévoir des efforts très importants avec des objets de dimensions à peine plus grandes; par exemple, on peut obtenir à une profondeur double, c'est-à-dire $1,20 \mathrm{~m}$, un ancrage provisoire de $80 \mathrm{kN}(8 \mathrm{t})$ avec un coefficient de sécurité de 1,5 , en utilisant un dispositif très rudimentaire pour sa mise en place.

\section{Références Bibliographiques}

Bureau Véritas (1975), « Règlement pour les navires en acier ", Section 4-3 et section 25-9, Bureau Véritas, Paris.

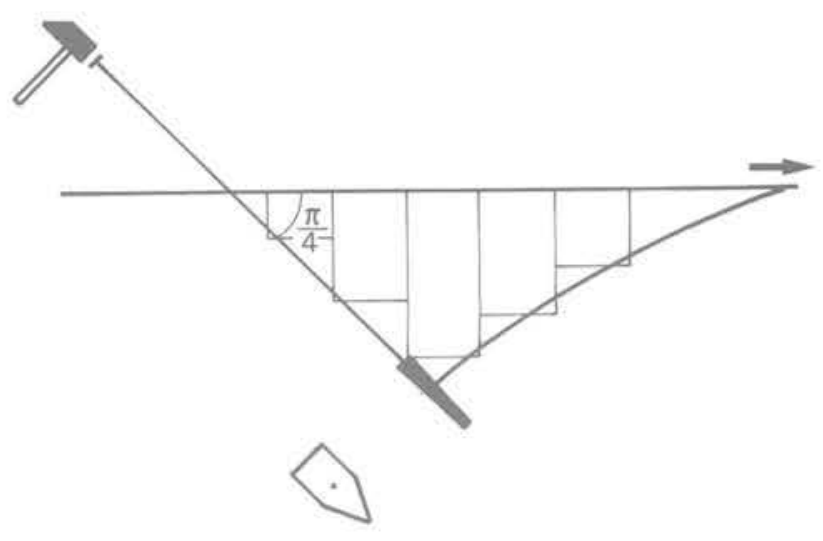

Fig. 28 Ancrage à terre

J. LE ROY (1972), Cours de Génie Maritime, ENSTA, première partie : Mouillage-Amarrage, p. 17-43.

J. MANDEL (1962), "Essais sur modèles réduits en Mécanique des terrains. Étude des conditions de similitude », Revue de I'Industrie Minérale, Sept. 1962, vol. $44, n^{\circ} 9$.

A. PUECH, J. MEUNIER et M. PAILLARD (1978), "Behaviour of anchors in different soil conditions ", $X^{\text {th }}$ Offshore Technology Conference, Houston, Mai 1978. 